\title{
Neurally Mediated Syncope Prediction Based on Changes of Cardiovascular Performance Surrogates
}

\author{
Algorithms Comparison
}

\author{
R. Couceiro, P. Carvalho, R. P. Paiva \\ and $\mathrm{J}$. Henriques \\ Department of Informatics Engineering \\ University of Coimbra \\ Coimbra, Portugal
}

\author{
J. Muehlsteff \\ Philips Research Laboratories Europe \\ Eindhoven, Netherlands
}

\author{
C. Eickholt, C. Brinkmeyer M. Kelm, C. \\ Meyer \\ Division of Cardiology, Pneumology, and \\ Angiology \\ Heinrich-Heine \\ University Hospital \\ Dusseldorf, Germany
}

\begin{abstract}
Two methodologies for neurally mediated syncope (NMS) prediction, based on the joint analysis of the electrocardiogram (ECG) and photoplethysmogram (PPG), are compared. Several features that characterize the variations in the inotropic, chronotropic, vascular tone and blood pressure surrogates were extracted and fed into two prediction models. The first method is based on the combination of the Minkowski distance metric with a threshold-based approach to evaluate the changes in the extracted features regarding the patient orthostatic stable state. The second method implements a SVM classification model to identify pre-syncope events. The output of the classification model is regularized using a "Firing power" (FP) measure and a threshold-based approach is used to generate alarms. Both methodologies were validated in 43 subjects using a three-way data split approach. The results achieved by the presented methodologies show that the first methodology is able to predict syncope episodes with better accuracy (sensitivity (SE) of $100 \%$ and specificity (SP) of $92 \%$ ) while maintaining a low rate of false alarms (FPRh: $0.146 h^{-1}$ ) and good prediction time (aPTime: 217.58s).
\end{abstract}

Keywords- Photoplethysmography, electrocardiography, syncope, chronotropy, inotropy, cascular tone, minkowski distance support vector machine

\section{INTRODUCTION}

Syncope, more commonly known as fainting or passing out, is defined as a transient loss of consciousness caused by a reduction of blood flow to the brain. It is characterized by a rapid onset, short duration and spontaneous complete recovery [1]. Also referred as reflex syncope, neurally mediated syncope (NMS) accounts for $21 \%$ of all syncope episodes [1].

Syncope has a great socioeconomic and medical impact in our society. It is responsible for an annual total cost of \$1.7-2.4 billion in hospitalizations, which is equivalent to diseases such as asthma, HIV, and chronic obstructive pulmonary disease [2]. Moreover, with a rising of incidence as age advances, from 2.6 to 19.5 per 1000 person-years between 20 and above 80 years old $[3,4]$, syncope strongly affects the life quality of elderly population. The risk of injuries, increased rates of hospitalization and loss of independency are common consequences within the elderly.

This work was supported by EU projects HeartCycle (FP7-216695), CISUC (Center for Informatics and Systems of University of Coimbra), iCIS (CENTRO-07-ST24-FEDER-002003), Welcome (FP7-ICT-2013-10) and HeartSafe (PTDC-EEI-PRO-2857-2012).
NMS results from an intermittent and inappropriate occurrence of cardiovascular reflex mechanisms, with orthostatic stress being one of the most common triggers. The excessive stimulation of a hypersensitive autonomic nervous system, results in a paradoxical withdrawal in the sympathetic tone leading to cardioinhibition, hypotension due to vasodepression and consequently to a loss of consciousness [5, $6]$.

In the later decades several algorithms for the prediction of syncope episodes based on the analysis of variations of heart rate (HR) and systolic blood pressure have been proposed [7, 8]. More recently, the analysis of the photoplethysmogram (PPG) for the extraction of blood pressure dependent parameters caught the attention of researchers. Several authors adopted pulse arrival time (PAT) has surrogate for SBP changes during impending syncope [9-11]. In our previous work [6], several parameters were analyzed in order to characterize possible mechanisms underlying the development of NMS.

In the current paper, we present a comparison between two methodologies for syncope prediction, which are based on the evaluation of several cardiovascular parameters extracted from the joint analysis of the electrocardiogram (ECG) and photoplethysmogram (PPG). Surrogates of the chronotropic (heart rate - HR), inotropic (left ventricular ejection time LVET), vascular tone and blood pressure (PAT, stiffness index - SI - and reflection index - SI, respectively) are extracted and normalized, leading to the definition of ten features, from which seven features were selected. In the first methodology, a simple, highly interpretable and easily implementable approach in embedded systems was adopted. Here, the distance to the orthostatic reference was calculated using the Minkowski distance metric and a threshold-based approach was adopted to detect impending syncope. In the second methodology, a complex, although highly recognized pattern recognition method was adopted. The selected features are fed into a support vector machine (SVM) in order to detect the onset of a pre-syncope event. The binary output of the classification model is regularized using a firing power measure [12] and a 
TABLE I. PATIENT CHARACTERISTICS (AVG \pm STD)

\begin{tabular}{ccc}
\hline \hline & $\begin{array}{c}\text { Tilt positive } \\
(\# 21)\end{array}$ & $\begin{array}{c}\text { Tilt negative } \\
(\# 22)\end{array}$ \\
\hline Age [y] & $57 \pm 18$ & $63 \pm 17$ \\
Weight [kg] & $86 \pm 15$ & $74 \pm 13$ \\
BMI [kg/m2] & $27.1 \pm 4.6$ & $26 \pm 5$ \\
Male/female & $13 / 8$ & $10 / 12$ \\
GTN yes/no & $15 / 6$ & $15 / 7$
\end{tabular}

threshold-based approach was adopted to detect impending syncope.

The remainder of the paper is organized as follows. In section II, the data collection and measurement protocol are described. The parameter extraction and syncope prediction methodologies are presented in section III. The results achieved by the two methodologies are compared and discussed in section IV and the main conclusions presented in section V.

\section{EXPERIMENTAL SETUP}

In the current study, 55 patients with unexplained syncope were enrolled for a diagnostic head-up tilt table tests (HUTT). In order to participate the present study (NCT01262508) all the patients gave a written informed consent.

The HUTT consisted in four steps. First, the patient is at supine position during a resting period of at least 15 minutes. Second, the patient is tilted to a passive standing position $\left(70^{\circ}\right)$ for about 20 minutes. Third, if no syncope occurred in the previous step, the patient remains more 15 minutes after the administration of min $400 \mu \mathrm{g}$ of glycerol trinitrate (GTN). Finally, the patient is tilted back to the supine position. At any of the before mentioned steps the patient is tilted back to supine position if syncope occurs. Prodromal symptoms such as dizziness, sweat and tremor were documented during the whole procedure.

According to the European Society of Cardiology guidelines [4], the test was classified as positive if syncope or pre-syncope occurs in the presence of bradycardia, hypotension, or both.

Due to BP regulation failures not caused by syncope, presence of arrhythmias and/or poor quality in BP and PPG signals, the data acquired from 12 patients was disregarded. In Table I we present the biometric characteristics of the patients involved in the present study.

The acquisition of the PPG and ECG signals was performed using a Philips MP50 patient monitor and stored on a laptop, while continuous (beat-to-beat) blood pressure (BP) was assessed using a "Taskforce Monitor" [12]. The data collected with both systems was synchronized using the ECG signal simultaneously acquired by both systems. Details of the acquisition system can be found in [6].

\section{METHODS}

The proposed methodologies resort on three main steps, which are: 1) Parameter extraction and post-processing; 2) Feature evaluation and; 3) Syncope prediction.

\section{A. Parameter extraction and post-processing}

TABLE II. CORRESPONDENCE BETWEEN PARAMETERS/FEATURES INDEXES AND NAMES

\begin{tabular}{cccc}
\hline \hline $\begin{array}{c}\text { Parameter } \\
\text { name }\end{array}$ & $\begin{array}{c}\text { Parameter } \\
\text { index }\end{array}$ & $\begin{array}{c}\text { Feature name } \\
\left(1^{\text {st }} \text { set }\right)\end{array}$ & $\begin{array}{c}\text { Feature name } \\
\left(2^{\text {nd }} \text { set }\right)\end{array}$ \\
\hline HR & $P R_{1}$ & $\widetilde{P R}_{1}$ & $\Delta P R_{1}$ \\
LVET & $P R_{2}$ & $\widetilde{P R}_{2}$ & $\Delta P R_{2}$ \\
SI & $P R_{3}$ & $\widetilde{P R}_{3}$ & $\Delta P R_{3}$ \\
RI & $P R_{4}$ & $\widetilde{P R}_{4}$ & $\Delta P R_{4}$ \\
PAT & $P R_{5}$ & $\widetilde{P R}_{5}$ & $\Delta P R_{5}$
\end{tabular}

A commonly observed mechanism during the onset of impending syncope is cardioinhibition, resulting from the increase of the parasympathetic activity, which can be assessed via HR. An algorithm similar to the approach discussed in [13] was used to detect the R-peaks in the ECG, which were used to extract the HR.

The increase in the contractility of the left ventricle, known to be a common trigger of NMS due to the excessive stimulation of the ventricular mechanoreceptors, was assessed via LVET using an algorithm similar to the proposed in [14].

The changes in vascular tone and blood pressure were assed from the analysis of the morphology of the PPG waveform, and joint analysis of the ECG and PPG signals. First, the stiffness index (SI) was defined by the time span between the forward and reflected waves of the PPG pulse. This index is associated with velocity of a pulse wave in large arteries [15] and correlates with pulse pressure [16]. Second, the reflection index (RI), which is correlated with small artery stiffness [15], was determined by the ratio between the amplitude of the reflected and forward waves of the PPG pulse. Finally, pulse arrival time $\left(\mathrm{PAT}_{80 \%}\right)$, which has been associated with changes in $\mathrm{BP}$ during impending syncope [11], was defined as the time span between the R-peak of the ECG and the time instant at the $80 \%$ amplitude of the correspondent PPG pulse. The characteristics points of the PPG waveform were assessed using the algorithm described in [14].

\section{B. Feature evaluation and selection}

PPG signals are often prone to several sources of errors, which can lead to the extraction of unreliable parameters. In order to avoid incorrect interpretations of the extracted parameters, an outlier removal approach similar to the one proposed in [17] has been adopted. In this approach, the main objective is to detect sporadic values that greatly differ from the parameters main trend using a sliding window boxplot analysis.

The extracted parameters were normalized leading to the definition of 10 features (presented in Table II). The first set of five features result from the normalization of the extracted parameters regarding the patients orthostatic stable state and were defined as:

$$
F T_{i}(t)=\widetilde{P R}_{i}(t)=\frac{P R_{i}(t)}{P \text { Rref }_{i}}, i=1, \ldots, 5
$$

where $F T_{i}$ is the $\mathrm{i}^{\text {th }}$ feature, $P R_{i}$ is the $\mathrm{i}^{\text {th }}$ parameter, $P R r e f_{i}$ is the average of each parameter during the second minute (reference window where orthostatic stabilization is achieved [18]) after the patient was tilted to the upright position and $t$ is the time instant. 
The second set of features results from the normalization of each parameter regarding to the last 1.5 minutes (minimum response time according to [11]), and was defined as:

$$
\begin{aligned}
F T_{i+5}(t)=\Delta P R_{i}(t) & =\frac{P R_{i}(t)-P R_{i}(t-1.5 \mathrm{~min})}{P R r e f_{i}} \\
, i & =1, \ldots, 5
\end{aligned}
$$

The feature selection step was completed using the approach proposed in [19] were the most suitable features for syncope prediction are selected based on the evaluation of a score metric (FSS), resultant from the analysis of each feature relevance - area under curve (AUC) of the receiver operand characteristic (ROC) curve - and redundancy - spearman's rank correlation coefficient (RCC). The FSS metric is defined as follows:

$$
F S S_{i}=\operatorname{AUC}\left(F T_{i}\right)-\frac{\left|\sum_{F T_{j} \in S} R C C\left(F T_{i}, F T_{j}\right)\right|}{|S|}
$$

where $S$ is the subset of selected features at each iteration and $|S|$ its cardinality. In sum, a total of seven features were selected, which are presented in a decreasing order (i.e. from the highest to the lowest FSS) as follows: 1) $\widetilde{P R}_{5}$;2) $\widetilde{P R}_{3} ; 3$ ) $\triangle P R_{1} ; \widetilde{P R}_{4} ; \Delta P R_{5} ; \triangle P R_{3}$ and $\triangle P R_{2}$.

\section{Syncope prediction}

\section{1) Methodology 1 overview}

The rationale behind the first methodology is that the abrupt variations observed in the extracted features, just before the onset of syncope, can be captured by analyzing the distance between each point in the feature space and the patient stable orthostatic reference, at the beginning of the standing period (FTref). These variations are illustrated in Figure 1, where it is possible to observe the development of the feature space trajectory in two patients (with/without NMS) during a HUTT.

The selection of the distance metric that better suits the syncope prediction problem was performed using a combination of a 5-fold cross-validation scheme with ROC analysis, for the following distance metrics: 1) Euclidean; 2) standardized Euclidean; 3) Cityblock; 4) Minkowski; 5) Chebychev; 6) Mahalanobis and; 7) Cosine. The Minkowski distance metric achieved the best results and therefore was selected to calculate the distance to a stable orthostatic reference, as presented in eq. (4).

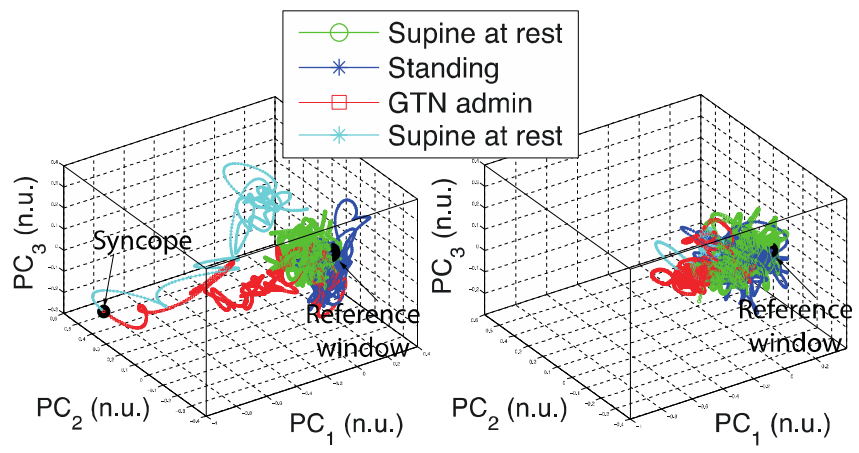

Figure 1. Illustration of the trajectory of the three principal components of a principal component analysis (PCA) extracted from the most discriminative features, during HUTT procedure. Left: 69-year-old patient with manifested syncope and GTN provocation. Right: 78-year-old patient with no syncope
Feature variations that were identified has not being associated with impending syncope were eliminated, i.e. $\widetilde{P R}_{3,5}$ values above unit and $\widetilde{P R}_{4}$ values bellow unit were set to one. Furthermore, $\triangle P R_{1}$ values bellow zero and $\triangle P R_{2,3,5}$ values above zero were set to zero.

$$
F D(t)=\left(\sum_{i=1}^{7}\left|F T_{i}(t)-F \operatorname{Tr} e f_{i}\right|\right)^{1 / p}, p=0.707
$$

where $F D(t)$ is the Minkowski distance at the time instant $t$.

Impending NMS was detected when FD crosses a predefined optimal threshold $\left(\mathrm{TH}_{\mathrm{O}}=3.256\right)$. The SBP, FD and HUTT sequence are presented in Figure 2 for an example case of 69-year-old patient.

\section{2) Methodology 2 overview}

In second methodology a support vector machine (SVM) classification model was adopted for the identification of presyncope events. The classification process was performed using the algorithm C-Support Vector Classification (C-SVC) algorithm [20], with a radial basis function kernel. To determine the SVM parameters, cost $(C)$ and gamma $(\gamma)$, a grid-search method using 5-fold cross-validation was used. The cross-validation process was repeated with groups of exponentially growing gamma/cost pairs $(C=$ $2^{-5}, 2^{-3}, \ldots, 2^{5}$ and $\left.\gamma=2^{-10}, 2^{-13}, \ldots, 2^{10}\right)$.

Aiming the reduction of false alarms the SVM binary output was regularized using the firing power (FP) method proposed by Teixeira et al. [12]. Here, the number of samples classified as pre-syncope were calculated over a 90 seconds sliding window (the minimum response time according to [11]). An alarm is generated when the regularized output of the SVM classifier crosses a predefined optimal threshold $\left(\mathrm{FP}_{0}=0.39\right)$.

\section{RESULTS AND DISCUSSION}

The performance of the presented methodologies was evaluated using a three-way data split scheme, where the dataset is randomly partitioned into train/validation (30

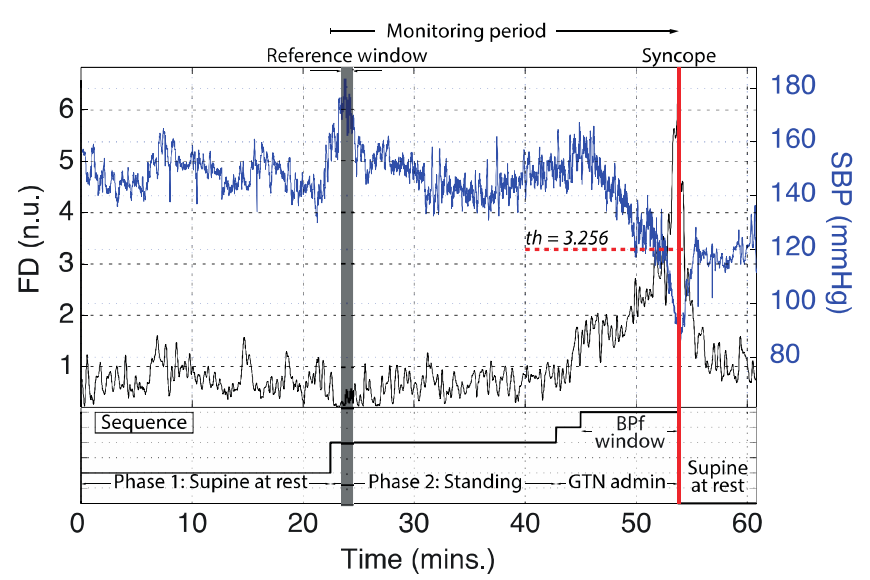

Figure 2. HUTT of a 69-year-old patient with manifested syncope and GTN provocation. Top diagram: SBP (blue) and FD (black) time series during HUTT. Bottom diagram: Phases of HUTT. Reference window represent as a black bar, corresponding to the second minute of phase 2. BPf window corresponds time between the start of BP fall and the syncope episode. 
patients) and test (13 patients) subsets.

The feature and model selection steps were evaluated with the train/validation subset using a 5-fold cross validation (5f$\mathrm{CV}$ ) approach (repeated 20 times), while the real performance of the presented methodologies was evaluated using the test subset.

The average and standard deviation (avg \pm std) of the following metrics were used to evaluate the performance of the presented methodologies: F-measure (F-m), sensitivity (SE) and specificity (SP), positive predictive value (PPV), false positive rate per hour (FPRh), prediction time average (aPTime) and standard deviation (sPTime). A true positive (TP) was considered if an alarm is generated within the BPf window (time span between the onset of BP decrease and the syncope episode) and a false positive was considered otherwise. A true negative (TN) was considered if no alarms were generated outside the BPf window, while a false negative (FN) was assigned in case of alarms are generated within this time frame. The FPRh was defined as the ratio between the number of false alarms (i.e. false positives) and the total length of the non-BPf windows (in hours). The PTime was defined as the time span between the first alarm and the syncope episode.

The performance of both methodologies was evaluated in two separate phases. First, both algorithm performances were evaluated using a 5 -fold cross validation approach (repeated 20 times) using the train/validation subset. Additionally, the selection of the best SVM classification model and the determination of the optimal thresholds for both methodologies were also performed during this phase.

In order to select the best SVM model and to avoid over fitting, the best five models, corresponding to the 5-fold SVM iteration achieving the best average performance, were selected and the mean of their outputs was calculated and regularized using the firing power method.

The optimal threshold for the first methodology was defined as $T H_{0}=3.256$, while for the second methodology the optimal firing power needed to trigger an alarm was defined as $\mathrm{FP}_{0}=0.39$.

Table III summarizes the best results achieved by the two methodologies during both train/validation and test phases.

The first methodology achieved a SE of $89 \%$, a SP of $96.7 \%$ and a PPV of $94.42 \%$ during the train/validation phase. Additionally, a low FPRh $0.18 \mathrm{~h}^{-1}$ and an aPTime of $65.37 \mathrm{~s}$ were also obtained. These results were consolidated in the test phase, where a SE of $100 \%$, a SP of $92.3 \%$ and a PPV of $85.7 \%$ were reached. Moreover, the number of false positives per hour is low (FPRh: $0.146 \mathrm{~h}^{-1}$ ) and a good prediction time was achieved (aPTime: 217.58s).

Contrarily, the second methodology presented a lower performance in both train/validation and test phases. In the train/validation, a SE of $92.3 \%$, a SP of $91.7 \%$ and a PPV of $87.4 \%$ were achieved, which represents a decrease of approximately $3 \%$ in the algorithms F-measure score, comparatively to the first methodology. Additionally, there was an increase of $0.09 \mathrm{~h}^{-1}$ in the false positive rate, followed by an increase in the prediction time of approximately $5 \mathrm{~s}$. In the test
TABLE III. PERFORMANCE OF THE PRESENTED ALGORITHMS IN THE VALIDATION $(F D V A L)$ AND TEST (FDTS) PHASES

\begin{tabular}{|c|c|c|c|c|c|c|c|}
\hline Phase & $\begin{array}{c}\text { Score }^{\dagger} \\
(\%)\end{array}$ & $\begin{array}{l}\text { SE } \\
(\%)\end{array}$ & $\begin{array}{l}\text { SP } \\
(\%)\end{array}$ & $\begin{array}{l}\text { PPV } \\
(\%)\end{array}$ & $\begin{array}{c}\text { FPRh } \\
\left(h^{-1}\right)\end{array}$ & $\begin{array}{c}\text { aPTime } \\
(\mathrm{s})\end{array}$ & $\begin{array}{l}\text { sPTime } \\
\text { (s) }\end{array}$ \\
\hline$F D v a l^{*}$ & $91.8 \pm 2$ & $89 \pm 3.3$ & $96.7 \pm 0$ & $94.42 \pm 0.8$ & $0.18 \pm 0.05$ & $65.37 \pm 2.6$ & $40.6 \pm 5.08$ \\
\hline MI FDts & 92.3 & 100 & 92.3 & 85.7 & 0.146 & 217.58 & 197.45 \\
\hline$F D v a l^{*}$ & $88.6 \pm 3$ & $92.3 \pm 5$ & $91.7 .3 \pm 2$ & $87.4 \pm 3$ & $0.27 \pm 0.11$ & $70.6 \pm 5.2$ & $59.2 \pm 8.5$ \\
\hline${ }^{M 2}$ FDts & 92.3 & 100 & 92.3 & 85.7 & 0.146 & 161.08 & 218.71 \\
\hline
\end{tabular}

phase, similar results were achieved, with the exception to a decrease in the prediction time to $161.08 \mathrm{~s}$.

\section{CONCLUSIONS AND FUTURE WORK}

In the current paper a comparison between two methodologies for prediction of impending syncope was presented. Both methodologies resort on the joint analysis of ECG and PPG signals, for the assessment of parameters strongly associated with the development of impending syncope. The first methodology is based on the assessment of a single distance measure and a threshold based approach to detect NMS. In the second methodology, an SVM classification model in used to detect the pre-syncope events. The binary output of the model is regularized using a firing power (FP) measure and a threshold-based approach is used to generate alarms.

The presented methodologies were evaluated on a dataset composed by 43 patients (21 patients with syncope episodes) using a three-way data split approach. The results achieved by the two methodologies show that the first methodology is capable predicting impending syncope more accurately, presenting a best performance during both training/validation and test phases.

Future work will focus on the further evaluation of distance metrics such as the Mahalanobis distance, where the contribution of the extracted features to feature distance metric will be weighted according to the relevance of each feature to the syncope prediction problem.

\section{ACKNOWLEDGMENT}

We thank the medical and nursing staff of the Neurocardiology Unit, Division of Cardiology, Pneumology and Angiology, University $\mathrm{Du}$ sseldorf, for supporting this study. We thank especially Sandy Gläser for excellent technical support.

\section{REFERENCES}

A. Moya, R. Sutton, F. Ammirati, J. J. Blanc, M. Brignole, J. B. Dahm, et al., "Guidelines for the diagnosis and management of syncope (version 2009)," Eur Heart J, vol. 30, pp. 2631-71, Nov 2009.

C. Kessler, J. M. Tristano, and R. De Lorenzo, "The Emergency Department Approach to Syncope: Evidence-based Guidelines and Prediction Rules," Emergency Medicine Clinics of North America, vol. 28, pp. 487-500, 8// 2010.

E. S. Soteriades, J. C. Evans, M. G. Larson, M. H. Chen, L. Chen, E. J. Benjamin, et al., "Incidence and Prognosis of Syncope," New England Journal of Medicine, vol. 347, pp. 878-885, 2002.

N. Colman, K. Nahm, K. S. Ganzeboom, W. K. Shen, J. Reitsma, M. Linzer, et al., "Epidemiology of reflex syncope," Clin Auton Res, vol. 14 Suppl 1, pp. 9-17, Oct 2004. 
[5] D. M. Lemonick, "Evaluation of Syncope in the Emergency Department," American Journal of Clinical Medicine, vol. 7, 2010.

[6] R. Couceiro, P. Carvalho, R. P. Paiva, J. Muehlsteff, J. Henriques, V. Schulze, et al., "Characterization of surrogate parameters for blood pressure regulation in neurally-mediated syncope," in 35th Annual Int. Conf. of the IEEE Eng. in Medicine and Biology Society, EMBC 2013, 2013, pp. 5381-5385.

[7] N. Virag, R. Sutton, R. Vetter, T. Markowitz, and M. Erickson, "Prediction of vasovagal syncope from heart rate and blood pressure trend and variability: Experience in 1,155 patients," Heart Rhythm, vol. 4, pp. 1375-1382, 2007.

[8] C. Chun-An, C. Hsin, and C. Hung-Wen, "Early detection of vasovagal syncope in tilt-up test with hemodynamic and autonomic study," in Computing in Cardiology, 2011, 2011, pp. 529-532.

[9] J. Muehlsteff, A. Ritz, T. Drexel, C. Eickholt, P. Carvalho, R. Couceiro, et al., "Pulse Arrival Time as surrogate for systolic blood pressure changes during impending neurally mediated syncope," in 34th Annual Int. Conf. of the IEEE Eng. in Medicine and Biology Society, EMBC 2012, 2012, pp. 4283-4286.

[10] C. Meyer, G. Morren, J. Muehlsteff, C. Heiss, T. Lauer, P. Schauerte, et al., "Predicting neurally mediated syncope based on pulse arrival time: algorithm development and preliminary results," J Cardiovasc Electrophysiol, vol. 22, pp. 1042-8, Sep 2011.

[11] J. Muehlsteff, T. Correia, R. Couceiro, P. Carvalho, A. Ritz, C. Eickholt, et al., "Detection of hemodynamic adaptations during impending syncope: Implementation of a robust algorithm based on pulse arrival time measurements only," 35th Annual Int. Conf. of the IEEE Eng. in Medicine and Biology Society, EMBC 2013, vol. 2013, pp. 2291-4, 2013.

[12] C. Teixeira, B. Direito, M. Bandarabadi, and A. Dourado, "Output regularization of SVM seizure predictors: Kalman Filter versus the "Firing Power" method," in 34th Annual Int. Conf. of the IEEE
Eng. in Medicine and Biology Society, EMBC 2012, 2012, pp. 6530-6533.

[13] Y. Sun, K. Chan, and S. Krishnan, "Characteristic wave detection in ECG signal using morphological transform," $B M C$ Cardiovascular Disorders, vol. 5, p. 28, 2005.

[14] R. Couceiro, P. Carvalho, R. P. Paiva, J. Henriques, M. Antunes, I. Quintal, et al., "Multi-Gaussian fitting for the assessment of left ventricular ejection time from the Photoplethysmogram," in EMBC2012, San Diego, 2012.

[15] S. S. DeLoach and R. R. Townsend, "Vascular Stiffness: Its Measurement and Significance for Epidemiologic and Outcome Studies," Clinical Journal of the American Society of Nephrology, vol. 3, pp. 184-192, 2008.

[16] M. Baruch, D. Warburton, S. Bredin, A. Cote, D. Gerdt, and C. Adkins, "Pulse Decomposition Analysis of the digital arterial pulse during hemorrhage simulation," Nonlinear Biomedical Physics, vol. 5 , p. 1, 2011

[17] O. Salem, L. Yaning, and A. Mehaoua, "A lightweight anomaly detection framework for medical wireless sensor networks," in Wireless Communications and Networking Conference (WCNC), 2013 IEEE, 2013, pp. 4358-4363.

[18] B. P. Grubb, "Pathophysiology and differential diagnosis of neurocardiogenic syncope," The American Journal of Cardiology, vol. 84, pp. 3-9, 1999.

[19] R. Wang and K. Tang, "Feature Selection for Maximizing the Area Under the ROC Curve," in Data Mining Workshops, 2009. ICDMW '09. IEEE International Conference on, 2009, pp. 400405.

[20] C.-C. Chang and C.-J. Lin, "LIBSVM : a library for support vector machines. ACM Transactions on Intelligent Systems and Technology," vol. 2, pp. 27:1--27:27, 2011. 